\title{
Aspectos epidemiológicos dos acidentes ofídicos ocorridos no estado do Piauí, Nordeste do Brasil, entre os anos de 2003 e 2017
}

\author{
Sâmia Caroline Melo Araújo ${ }^{1}$ (1) \& Etielle Barroso de Andrade $^{2}$ ()
}

(1) Faculdade Evangélica do Meio Norte, Programa de Pós-Graduação em Ciências Ambientais e Saúde, Rua Valença 545, Tabuleta 64018-535, Teresina, Piauí, Brasil. E-mail: carolinesamia93@gmail.com

(2) Instituto Federal do Piauí - Campus Pedro II, Grupo de Pesquisa em Biodiversidade e Biotecnologia do Centro-Norte Piauiense - BIOTECPI, Rua Antonino Martins de Andrade 750, Engenho Novo, Pedro II 64255-000, Piauí, Brasil. E-mail: etlandrade@hotmail.com

Araújo S.C.M. \& Andrade E.B. (2019) Aspectos epidemiológicos dos acidentes ofídicos ocorridos no estado do Piauí, Nordeste do Brasil, entre os anos de 2003 e 2017. Pesquisa e Ensino em Ciências Exatas e da Natureza, 3(2): 154-165. http://dx.doi.org/10.29215/pecen.v3i2.1265

Editor acadêmico: Arielson dos Santos Protázio. Recebido: 26 Março 2019. Aceito: 08 Outubro 2019. Publicado: 14 Outubro 2019.

Resumo: A região Nordeste possui o terceiro maior coeficiente médio de acidentes ofídicos do país, contudo, os perfis epidemiológicos e clínicos dos casos são ainda bastante escassos. Assim, o presente estudo tem como objetivo analisar os aspectos epidemiológicos e o coeficiente de incidência dos acidentes com serpentes ocorridos no estado do Piauí, no período de 2003 a 2017. As informações sobre as ocorrências dos acidentes ofídicos nos municípios do Piauí e as variáveis epidemiológicas foram coletadas no Sistema de Informação de Agravos de Notificação, tendo como base de busca os anos de 2003 a 2017. As informações demográficas para calcular o coeficiente de incidência de cada município foram coletadas no Instituto Brasileiro de Geografia e Estatística. Foram notificados um total de 3337 casos de acidentes ofídicos, dos quais 2334 ocorreram com serpentes peçonhentas. A ocorrência de casos foi maior com o sexo masculino e a faixa etária de maior envolvimento foi de 20 a 39 anos. A maioria dos casos apresentou sintomatologia leve evoluindo para a cura completa, com o período de atendimento após o acidente de até três horas. As serpentes que mais causaram acidentes foram as do gênero Bothrops. Dos municípios piauienses, os que apresentaram maiores coeficientes médios de incidência foram Lagoa do Barro do Piauí, Dom Inocêncio e Capitão Gervásio Oliveira, todos localizados na região sudeste do estado. O levantamento desses dados mostra a escassez de estudos sobre esses acidentes no estado do Piauí e a importância do preenchimento correto das fichas de notificação para o desenvolvimento de ações efetivas por parte das autoridades competentes.

Palavras chave: Acidentes ofídicos, SINAN, Bothrops, IBGE.

Epidemiological aspects of snake bite accidents in the state of Piauí, Northeast Brazil, between 2003 and 2017

Abstract: The Northeastern Brazilian region has the third highest average rate of snake bite accidents in the country, however, the epidemiological and clinical profiles of the cases are still scarce. Thus, the present study aims to analyze the epidemiological aspects and the incidence coefficient of snake bites occurring in the state of Piauí, from 2003 to 2017. Information on the occurrence of snake bite accidents in the municipalities of the state of Piauí and epidemiological variables was collected in the Information System for Notifiable Diseases, in the period from 2003 to 2017. Demographic information to calculate the incidence coefficient of each municipality was collected at the Brazilian Institute of Geography and Statistics. A total of de 3337 cases of snake bites have been reported, of which 2334 occurred with venomous snakes. The highest occurrence of the cases was observed among males and the age group with the greatest involvement was 20 to 39 years old. Most cases presented mild symptomatology progressing to 
complete cure, with the post-accident care period of up to three hours. The snakes that caused the most accidents were those of the genus Bothrops. Of the Piauí municipalities, those with the highest average incidence coefficient were Lagoa do Barro do Piauí, Dom Inocêncio and Capitão Gervásio Oliveira, all located in the southeastern region of the state. The present study reveals a lack of data on snake bites in the state of Piauí and importance of correctly completing the notification forms for the development of effective actions by the competent authorities.

Key words: Snake bites, SINAN, Bothrops, IBGE.

\section{Introdução}

Envenenamentos causados por serpentes peçonhentas são um grave problema de saúde pública em diversas partes do mundo, principalmente em países tropicais e em desenvolvimento, onde há uma grande diversidade de serpentes convivendo próximo das populações rurais e onde os sistemas de notificação são mais escassos (Pinho et al. 2004; Albuquerque et al. 2005; Waldez \& Vogt 2009; Gutiérrez 2011). A invasão dos habitats e substituição da cobertura vegetal natural por áreas plantadas ou pastagens e a precariedade dos serviços de saúde pública como, por exemplo, a dificuldade no atendimento e escassez de antivenenos específicos, são os principais fatores associados à elevada taxa de morbimortalidade dos acidentes ofídicos no mundo (Pinho \& Pereira 2001). Além disso, o número de vítimas que sobrevivem e ficam com sequelas físicas e psicológicas, devido à ação do veneno nos tecidos, pode ser mais elevado que o número de óbitos (Gutiérrez et al. 2006).

O número exato de acidentes com serpentes que ocorrem no mundo é bastante difícil de predizer. A Organização Mundial de Saúde (Brasil 2009) estima que ocorra mais de 500 milhões de acidentes com serpentes no mundo, sendo cerca de 2.7 milhões de envenenamento por serpentes peçonhentas, resultando em 20 a 100 mil mortes por ano. A mortalidade causada por serpentes peçonhentas varia de acordo com as diversas regiões do mundo (Gutiérrez et al. 2007). Nos países desenvolvidos da Europa, nos Estados Unidos e Canadá, os acidentes são bastante raros, ocorrendo apenas oito mil casos de envenenamento com serpentes peçonhentas por ano, sendo em média 35 casos fatais, o que leva a uma taxa de mortalidade de apenas $0.3 \%$ (Pinho \& Pereira 2001).

Contudo, a morbimortalidade dos acidentes ofídicos está intimamente relacionada com a pobreza. Na Ásia, estima-se que os acidentes com serpentes provoquem cerca de 15 a 55 mil mortes por ano, e na África, dos 500 mil casos de acidentes registrados, apenas $40 \%$ são hospitalizados e cerca de 30 mil pessoas vão à óbito todo ano (Harrison et al. 2009). Esses números podem ser ainda mais preocupantes, na Nigéria, por exemplo, a taxa de mortalidade chega a atingir $\mathbf{1 0 \%}$ e acredita-se que esse número seja ainda maior devido à subnotificação no país (Pinho et al. 2004).

$\mathrm{Na}$ América Latina, o Brasil apresenta o maior número de acidentes, com aproximadamente 22.000 casos e letalidade de cerca de $0.45 \%$ ao ano (Pinho et al. 2004; Lima et al. 2009), ocorrendo com maior frequência nas zonas rurais e regiões afastadas dos grandes centros urbanos (Albuquerque et al. 2005). Os primeiros registros de serpentes peçonhentas no Brasil datam do período colonial quando, em 1560, José de Anchieta fez observações clínicoepidemiológicas não sistematizadas de acidentes causados por jararacas, cascavéis e corais (Bochner \& Struchiner 2003, 2004; Lucas 2009). Contudo, os primeiros dados epidemiológicos foram registrados e publicados por Vital Brazil ao levantar o número de óbitos por serpentes ocorridos no estado de São Paulo entre os anos de 1897 e 1900, no qual analisou diversas variáveis, tais como sexo, idade da vítima, mês de ocorrência do acidente, local da picada, gênero da serpente, etc., que se constituíram a base dos atuais sistemas nacionais de informação e notificação (Bochner \& Struchiner 2003, 2004).

No Brasil, apesar da notificação obrigatória a partir de 1996 e do surgimento de vários sistemas de notificação e análise dos dados epidemiológicos (Sistema de Informação de Agravos de Notificação - SINAN, Sistema Nacional de Informações Tóxico-Farmacológicas - 
SINITOX/Fiocruz, Sistema de Informações Hospitalares do Sistema Único de Saúde e Sistema de Informações sobre Mortalidade - SIM) (Brito \& Barbosa 2012), os acidentes ofídicos continuam ainda bastante negligenciados pelas políticas públicas nacionais, principalmente no que diz respeito ao tratamento e a qualificação dos profissionais de saúde (Gutiérrez et al. 2006, 2007). Além disso, as dificuldades de acesso aos centros de saúde e a falta de registros nas regiões mais remotas do país gera um quadro de subnotificação que não reflete a real situação do problema, principalmente nas regiões Centro-Oeste, Norte e Nordeste do país (Brasil 2001).

Segundo o Ministério da Saúde (Brasil 2005), a região Nordeste possui o terceiro maior coeficiente médio de acidentes ofídicos do país (13 acidentes/100 mil hab.), contudo, os perfis epidemiológicos e clínicos dos casos são ainda bastante escassos. Poucos trabalhos sobre os acidentes ofídicos foram realizados na região Nordeste (Feitosa et al. 1997; Albuquerque et al. 2005; Casais-e-Silva \& Brazil 2009; Lemos et al. 2009; Lira-da-Silva et al. 2009; Brito \& Barbosa 2012; Saraiva et al. 2012; Albuquerque et al. 2013; Oliveira et al. 2015; Tavares 2016; Cunha et al. 2019).

No Piauí, a situação é ainda mais preocupante, já que há apenas três estudos abordando os casos de acidentes ofídicos no estado. Os primeiros foram feitos por Lira-da-Silva et al. (2009), no qual avaliaram a morbimortalidade dos acidentes ofídicos na região Nordeste e incluíram informações sobre os casos ocorridos no Piauí entre os anos 1999 e 2003. Cunha et al. (2019) avaliaram os casos de acidentes com animais peçonhentos no estado entre os anos 2007 a 2011 , onde relataram, dentre outros, os casos de ofidismo e o único trabalho realizado sobre o perfil epidemiológico dos acidentes com serpentes ocorridos exclusivamente no estado do Piauí foi feito por Oliveira et al. (2015), no qual analisaram dados referentes ao período de 2007 a 2012. No entanto, de lá para cá, pouco se sabe sobre a ocorrência de casos de acidentes com serpentes no estado. Dessa forma, o presente trabalho teve como objetivo analisar os aspectos epidemiológicos e o coeficiente de incidência dos acidentes ofídicos ocorridos no estado do Piauí, no período de 2003 a 2017.

\section{Metodologia}

Um estudo retrospectivo e quantitativo foi realizado utilizando dados sobre os acidentes ofídicos ocorridos no Estado do Piauí no período de 2003 a 2017. O Piauí, situado entre as coordenadas $02^{\circ} 44^{\prime}$ e $10^{\circ} 52^{\prime}$ de latitude Sul e entre $40^{\circ} 25^{\prime}$ e $45^{\circ} 59^{\prime}$ de longitude Leste, é considerado o terceiro maior dos nove estados que compõem a região Nordeste do Brasil (Figura 1), possuindo uma área territorial de $251.616 .823 \mathrm{~km}^{2}$. Segundo o Instituto de Geografia e Estatística (IBGE), possui uma população estimada em 3.264.531 habitantes para o ano de 2017.

O período analisado foi determinado de modo a oferecer um panorama dos acidentes com serpentes nos últimos 15 anos (2003 a 2017), preenchendo as lacunas de informação desde os registros mais antigo sobre a ocorrência de acidentes ofídicos, iniciados em 2003 (Lira-da-Silva et al. 2009; Oliveira et al. 2015; Cunha et al. 2019) até o ano em que estes dados foram disponibilizados no SINAN (2017). Dados mais recentes, referentes aos anos de 2018 e 2019, não puderam ser coletados, por não estarem disponíveis no sistema. Uma vez que os dados foram coletados através do sistema de notificação e sem identificação dos envolvidos nos acidentes, não houve a necessidade de recorrer ao Comitê de Ética.

As informações sobre as ocorrências dos acidentes ofídicos nos 224 municípios do estado foram coletadas junto ao Sistema de Informação de Agravos de Notificação (SINAN), utilizando como filtro "município de ocorrência", para registrar os casos reais de ocorrência por município, e o "tipo de serpente" para analisar apenas os casos de acidentes com serpentes.

Para a construção do perfil de distribuição dos acidentes ofídicos no estado, analisou-se as variáveis epidemiológicas básicas, tais como a sazonalidade dos casos durante o período analisado (2003-2017), sexo e faixa etária de maior ocorrência, a evolução do caso, a espécie de serpente envolvida no acidente, o tempo entre o acidente e o atendimento e a classificação clínica dos casos. As variáveis epidemiológicas seguiram os padrões abordados por Borges et al. (1999), Pinho et al. (2004), Bonan et al. (2010), entre outros. 
Para avaliar a distribuição dos casos de acidentes em relação ao número de habitantes de cada município foi calculado o coeficiente de incidência média dos acidentes ofídicos, no qual foi utilizado o número médio de acidentes registrados no período estudado, dividido pela média populacional residente no mesmo período e multiplicado por 100 mil habitantes. As informações demográficas de cada município foram coletadas junto ao Instituto Brasileiro de Geografia e Estatística-IBGE.
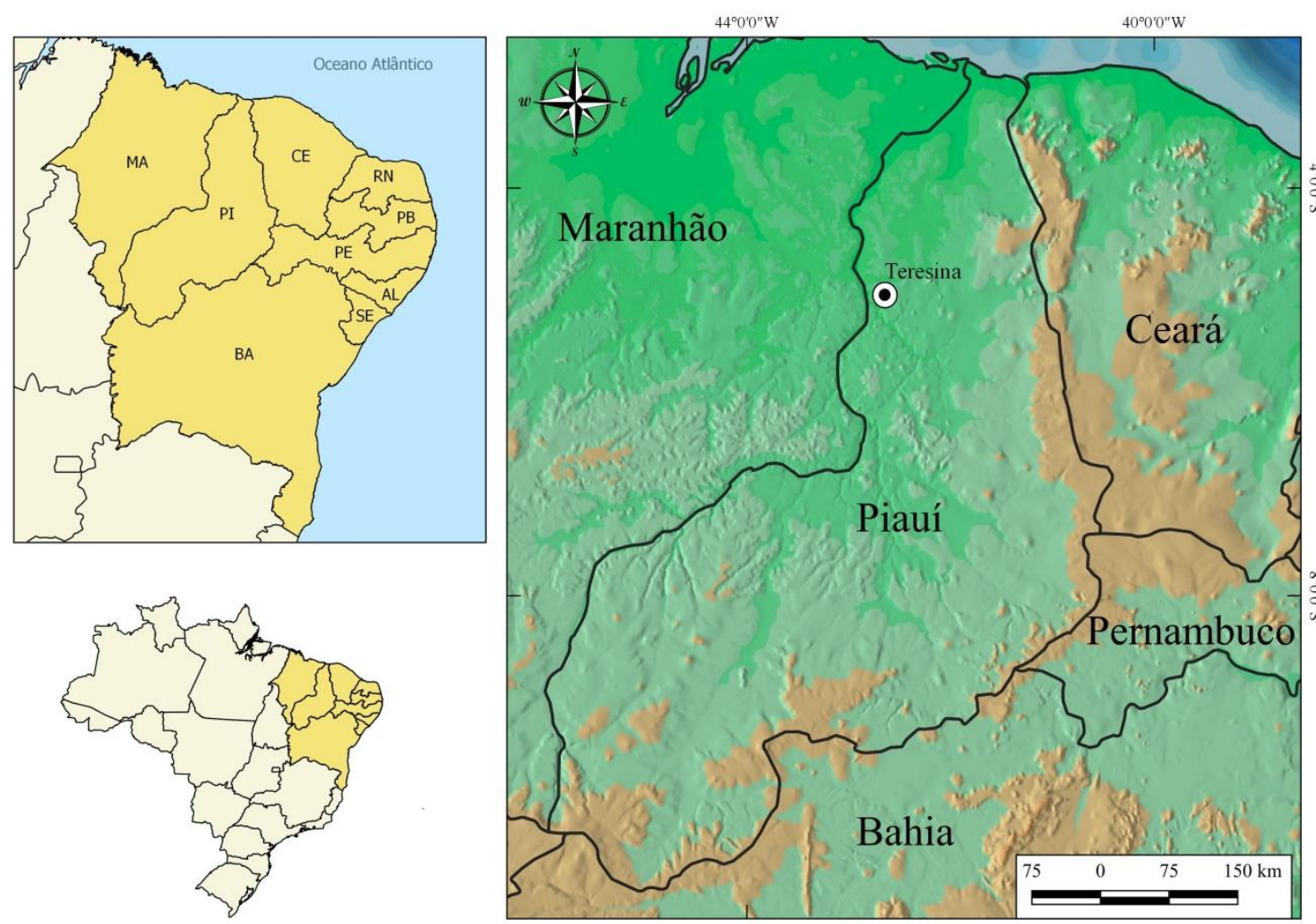

Figura 1. Mapa de localização geográfica do estado do Piauí, um dos nove estados que compõem a região Nordeste do Brasil, com destaque (círculo) para a capital Teresina.

\section{Resultados}

No período de 2003 a 2017 foram notificados no estado do Piauí 3337 casos de acidentes ofídicos, dos quais 2334 casos (cerca de 71\%) ocorreram com serpentes peçonhentas e 186 casos (5\%) com serpentes não peçonhentas, o que representa uma proporção de cerca de 16 vezes menos que o número de casos com serpentes peçonhentas. Os outros $25 \%$ dos casos (817 casos) não tiveram suas identificações concluídas, sendo registrados no sistema apenas como casos ignorados. O ano de 2006 apresentou maior ocorrência de casos seguido do ano de 2017, com 276 e 274 casos, respectivamente. O menor número de casos foi registrado no ano de 2015, com 167 casos (Figura 2).

Com relação ao sexo dos envolvidos nos acidentes, o sexo masculino foi mais afetado com $76.5 \%$ dos casos, o que representa uma taxa média de 170.2 casos por ano. $\mathrm{O}$ sexo feminino contou com apenas $23.5 \%$ dos casos, cerca de 52.3 casos por ano (Tabela 1). Os dados revelam uma grande prevalência dos acidentes envolvendo pessoas do sexo masculino, sendo registrado cerca de três vezes mais em relação ao sexo feminino.

A faixa etária com maior incidência dos casos foi aquela representada por indivíduos com idade variando entre 20 e 39 anos, representando cerca de $37 \%$ dos casos registrados, seguida do grupo formado por indivíduos com idade variando de 40 a 59 anos (28\% dos casos) (Tabela 2). Estes dois grupos compreendem jovens e adultos em idade produtiva e somam juntos mais de $64 \%$ do número de casos registrados. 


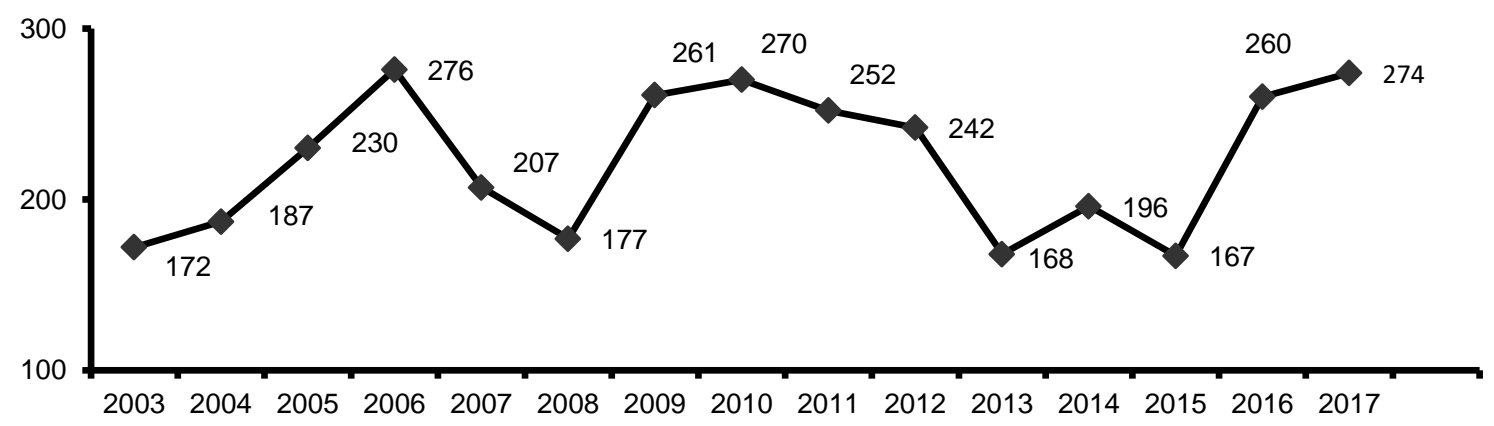

Figura 2. Números de acidentes com serpentes ocorridos no estado do Piauí, Nordeste do Brasil, entre os anos de 2003 e 2017.

Tabela 1. Acidentes com serpentes ocorridos no Piauí entre os anos de 2003 e 2017 de acordo com o sexo dos envolvidos.

\begin{tabular}{ccc}
\hline \multirow{2}{*}{ Ano } & \multicolumn{2}{c}{ Sexo } \\
\cline { 2 - 3 } & Masculino & Feminino \\
\hline 2003 & 131 & 41 \\
2004 & 136 & 51 \\
2006 & 173 & 58 \\
2007 & 220 & 56 \\
2008 & 163 & 44 \\
2009 & 133 & 44 \\
2010 & 213 & 48 \\
2011 & 216 & 54 \\
2012 & 197 & 55 \\
2013 & 181 & 60 \\
2014 & 127 & 41 \\
2015 & 145 & 51 \\
2016 & 124 & 41 \\
2017 & 187 & 73 \\
TOTAL & 207 & 67 \\
\hline
\end{tabular}

Tabela 2. Faixa etária dos casos de acidentes com serpentes no estado do Piauí no período de 2003 a 2017.

\begin{tabular}{cc}
\hline Idade & Número de casos \\
\hline Em branco/Ignorado & 1 \\
$<1$ ano & 40 \\
1 a 4 & 52 \\
5 a 9 & 138 \\
10 a 14 & 253 \\
15 a 19 & 321 \\
20 a 39 & 1227 \\
40 a 59 & 931 \\
60 a 64 & 138 \\
65 a 69 & 102 \\
70 a 79 & 110 \\
80 e + & 24 \\
Em branco/Ignorado & 1 \\
$<1$ ano & 40 \\
\hline
\end{tabular}

$\mathrm{Na}$ grande maioria dos casos, os envolvidos em acidentes com serpentes procuram atendimento médico rápido. $\mathrm{O}$ tempo decorrido entre $\mathrm{o}$ acidente e $\mathrm{o}$ atendimento médico foi, na grande maioria, de uma a três horas após o acidente (35\% dos casos). Os dados mostram também que o tempo de acidente/atendimento com grande ocorrência foi de três a seis horas $(22 \%$ dos casos) (Tabela 3). 
Tabela 3. Tempo decorrido entre o acidente e o atendimento dos acidentes com serpente no estado do Piauí entre os anos de 2003 e 2017.

\begin{tabular}{cc}
\hline Tempo de atendimento & Casos \\
\hline Ignorado/Branco & 303 \\
1 hora & 587 \\
$1-3$ horas & 1166 \\
$3-6$ horas & 706 \\
$6-12$ horas & 252 \\
$12 \mathrm{e}+$ horas & 323 \\
\hline
\end{tabular}

Em relação à gravidade dos casos, a maioria dos pacientes (56.8\% dos casos) que chegaram aos centros de saúde apresentaram quadros sintomáticos leves, cerca de $30 \%$ dos casos foram classificados como moderado e apenas $5 \%$ dos casos foram considerados graves. Cerca de $8.3 \%$ dos casos não tiveram seus quadros clínicos observados, sendo considerados apenas como ignorado no sistema (Tabela 4). Os indivíduos envolvidos nos acidentes com serpentes no estado do Piauí apresentaram boa evolução dos casos, sendo que cerca de $84.5 \%$ evoluíram para a cura completa, sem nenhum tipo de sequela. Contudo, em uma pequena parcela de casos o quadro evoluiu para cura, mas os pacientes apresentaram algum tipo de sequela decorrente da picada $(0.5 \%)$. Dado esse que foi observado apenas entre o período de 2003 a 2006, pois a partir de 2007 o sistema de notificação sofreu uma alteração, tendo o item "cura com sequela" retirado. Aproximadamente $1 \%$ dos casos apresentaram notificação de óbito decorrente da picada ou de alguma outra complicação (Tabela 4), o que representa uma taxa de cerca de 2.1 mortes por ano no Piauí.

Tabela 4. Quadro clínico e evolução final dos acidentes ofídicos ocorridos no estado do Piauí entre os anos de 2003 e 2017.

\begin{tabular}{ccc}
\hline Quadro clínico & Número de casos & $\%$ \\
\hline Ignorado/Branco & 278 & 8.3 \\
Leve & 1894 & 56.8 \\
Moderado & 997 & 29.9 \\
Grave & 168 & 5.0 \\
\hline Evolução do acidente & Número de casos & $\%$ \\
\hline Ignorado/Branco & 470 & 14.1 \\
Cura & 2821 & 84.5 \\
Cura com sequela & 16 & 0.5 \\
Óbito & 30 & 0.9 \\
Total de casos & 3.337 & \\
\hline
\end{tabular}

Dentre os mais de três mil casos de acidentes com serpente no estado do Piauí, as que provocaram maior número de acidentes foram as do gênero Bothrops, popularmente conhecida como jararaca, representando quase a metade do número total de casos (45\%), seguida do gênero Crotalus (21.2\%), popular cascavel. Juntos, os dois gêneros possuem grande importância médica, representando quase $70 \%$ dos casos de ofidismo no estado. As espécies dos gêneros Micrurus (coral verdadeira) e Lachesis (Surucucu-pico-de-jaca) tiveram pouca representação nos casos de acidentes, representando cerca de $2.4 \%$ e $0.3 \%$ dos casos, respectivamente (Tabela 5).

Tabela 5. Serpentes que mais causaram acidentes entre os anos 2003 e 2017 no estado do Piauí.

\begin{tabular}{cc}
\hline Serpentes & Número de casos \\
\hline Ignorado/Branco & 817 \\
Bothrops & 1533 \\
Crotalus & 709 \\
Micrurus & 81 \\
Lachesis & 11 \\
Não peçonhentas & 186 \\
\hline
\end{tabular}


Em relação aos casos notificados de acidentes entre os municípios do Piauí, observou-se uma divergência entre a ocorrência e o coeficiente médio de incidência. Os municípios que registraram a maior taxa de ocorrência média (soma das ocorrências de acidentes entre os anos de 2003 a 2017 divididos pelo número de anos pesquisados) foram Picos, Teresina e Dom Inocêncio, com 103, 85 e 82 casos/ano, respectivamente. Por outro lado, quando foi levado em consideração a população residente em cada município no período pesquisado, observou-se que municípios menores apresentaram maiores coeficientes médio de incidência. Os municípios que mais se destacaram foram Lagoa do Barro do Piauí (74.00/100.000 hab.), seguido de Dom Inocêncio (55.87/100.000 hab.) e Capitão Gervásio Oliveira (44.44/100.000 hab.), três municípios vizinhos localizados na região sudeste do estado. Outro munícipio que apresentou elevado coeficiente médio de incidência foi o município de Altos (43.34/100.000 hab.), localizado na região norte, próximo à capital Teresina (Figura 3).

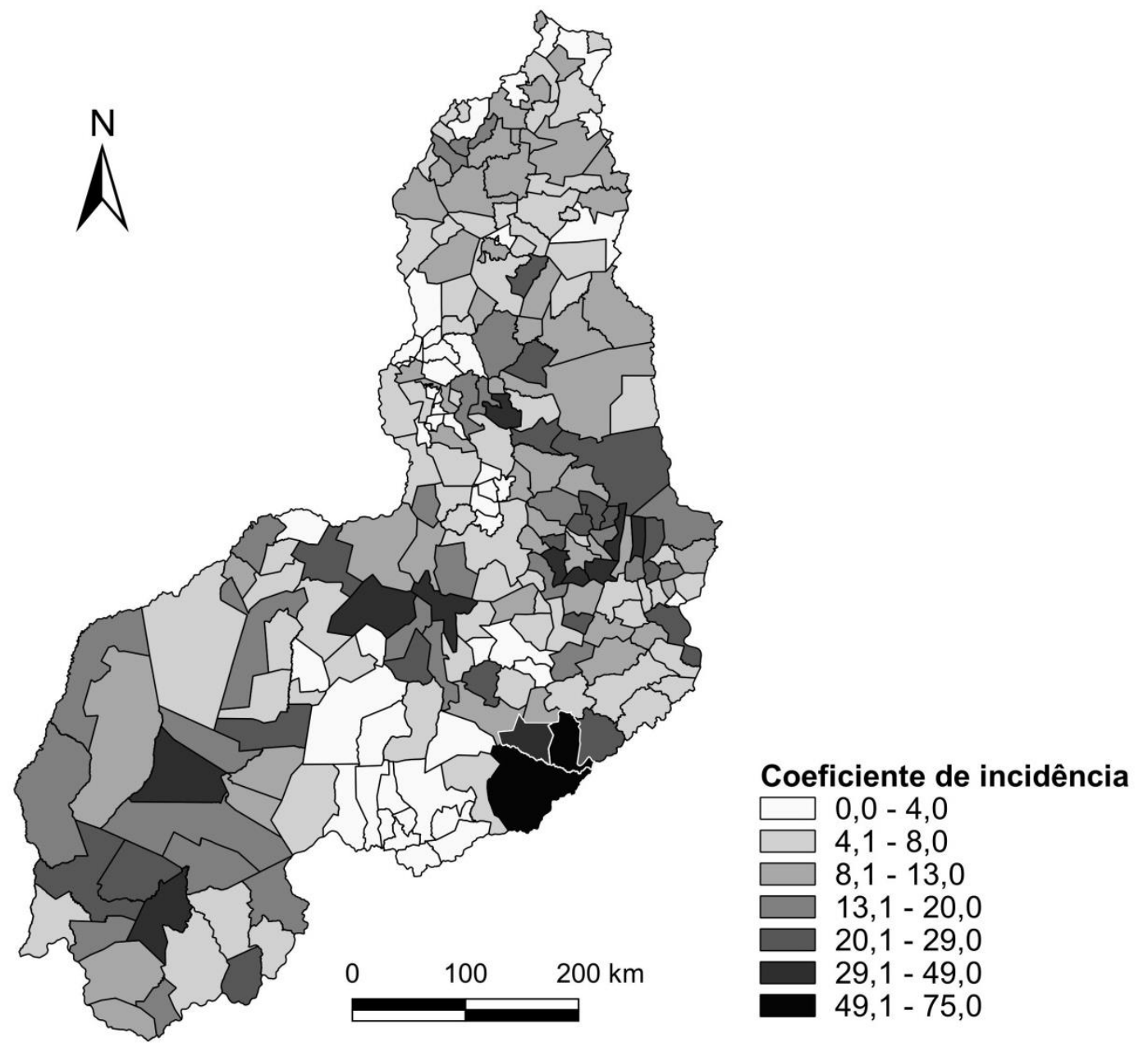

Figura 3. Coeficiente médio de incidência dos acidentes ofídicos ocorridos no estado do Piauí entre os anos de 2003 e 2017.

\section{Discussão}

Durante os 15 anos relativos à pesquisa (2003 a 2017) é possível observar um padrão temporal de variação dos acidentes com serpentes em torno de um número médio, no qual a cada quatro anos ocorre a elevação e posterior diminuição dos casos.

A elevada porcentagem do sexo masculino envolvidos com os acidentes ofídicos pode estar relacionada à maior atividade do homem fora do lar, seja por lazer ou por atividades no campo. $\mathrm{O}$ trabalhador agropecuário corre um maior risco de sofrer acidentes com serpentes, 
uma vez que, se encontra mais frequente na execução das atividades agrícolas, apresentando um maior contato com o hábitat desses animais (Ribeiro \& Jorge 1997; Bochner \& Struchiner 2003). Além disso, é comum a não utilização de equipamentos de proteção individual, deixando o trabalhador rural mais propício a esses acidentes. Estes dados corroboram outros estudos realizados no nordeste (Lira-da-Silva et al. 2009; Oliveira et al. 2015; Cunha et al. 2019) e em diversas regiões do Brasil, no qual a proporção dos acidentados é maior no sexo masculino uma vez que estes passam frequentemente mais tempo realizando atividades relacionadas ao campo (Bonan et al. 2010; D’Augustini et al. 2011; Sousa et al. 2013).

Os acidentes com serpentes estão mais relacionados à população rural, seja por residirem, por lazer ou trabalharem na zona rural (Rojas et al. 2007). Assim, os acidentes ofídicos são considerados acidentes de trabalho (Feitosa et al. 1997; Brito \& Barbosa 2012), uma vez que a ocorrência é frequente com pessoas que trabalham em atividade agrícola (Lemos et al. 2009). Além de serem tidos como acidentes de trabalho, os acidentes com serpentes se relacionam, ainda, com a urbanização das regiões afastadas do centro urbano, contribuindo na mistura do habitat desses animais com as atividades antrópicas (Rojas et al. 2007).

A faixa etária entre 20 e 39 anos apresenta maior concentração de pessoas trabalhando em atividades do campo, sendo uma idade de maior produtividade. Os resultados registrados aqui, no entanto, divergem dos dados coletados por Feitosa et al. (1997) em estudo realizado no estado do Ceará, no qual mostram que a faixa etária com mais ocorrência foi de 10 a 19 anos. Confronta, também, os dados de Albuquerque et al. (2005) coletados na Paraíba, onde afirmam que a faixa etária mais acometida foi de 10 a 39 anos. Por outro lado, os resultados apresentados aqui corroboram os dados de Saraiva et al. (2012) realizados também na Paraíba, onde a faixa etária de maior ocorrência foi 20 e 39 anos. Assim, apesar dos acidentes ocorrerem com maior frequência dentro da idade produtiva da população rural, principalmente do sexo masculino, a inconsistência dos dados reflete a falta de padronização na formação dos grupos etários.

A faixa etária de 40 a 64 anos, uma grande ocorrência de acidentes, o que se deve, provavelmente, ao maior número de idosos envolvidos em atividades rurais, possivelmente, para aumentar a renda familiar. No entanto, acima dos 65 anos ocorre um decréscimo em razão, provavelmente, da baixa atividade dessas pessoas na atividade agrícola. Pessoas com idade acima de 65 anos apresentam uma menor disposição para esses trabalhos pesados e que exigem muito esforço físico.

O tempo no atendimento é fundamental para evitar complicações futuras e até mesmo o óbito. Aqui, foi observado um atendimento de 1 a 3 horas após o acidente. Resultados semelhantes são registrados em outras localidades da região Nordeste (Saraiva et al. 2012) onde a dificuldade de acesso aos centros médicos pode ser um fator decisivo entre a cura e o agravamento do caso. Além disso, o lapso temporal de três a seis horas demonstra a demora por busca de atendimento em unidade de saúde, que pode ser devido ao uso de remédios medicinais alternativos na esperança de obter a cura. Deve-se também a longa distância do local do ocorrido para cidades com unidades competentes ou a transferência dos acidentados para um centro de atendimento mais adequado em outras cidades, já que o atendimento especializado no estado, geralmente, encontra-se na capital. Esses dados divergem de D’augustini et al. (2011) quando, em um estudo feito no estado de Santa Catarina, afirmam que o horário de maior atendimento foi com menos de uma hora do ocorrido. Mas, concordam com o trabalho de Oliveira et al. (2015) realizado no Piauí, onde o período de atendimento após a picada foi de até 3 horas.

A maior ocorrência de casos leves e a evolução para cura sem sequelas provavelmente demonstra a eficiência no tratamento com acidentes ofídicos, embora o maior ponto de referência no tratamento dos casos encontre-se na capital. Resultados semelhantes foram encontrados por Sousa et al. (2013) em um estudo realizado no município de Mossoró-Rio Grande do Norte, no qual classifica os casos leves como os que mais ocorrem (58.7\%), seguido dos moderados (27.7\%) e graves (11.2\%). Entretanto, contradizem os dados encontrados por Bernarde \& Gomes (2012) no trabalho desenvolvido no Acre, mostrando que predominaram os casos moderado, seguido dos leves. Apesar da maioria dos casos terem sido classificados como 
leves ou moderados, houve ainda muitos casos em que a classificação clínica foi ignorada (13.9\%), o que dificulta a realização de um prognóstico e a realização do tratamento adequado.

Os acidentes com serpentes ocorrem, em sua maioria, com o gênero Bothrops. Os dados coletados aqui corroboram os resultados de Rojas et al. (2007), no qual mostra o gênero Bothrops como causador do maior número de casos de acidentes ofídicos (65.7\%), seguido do gênero Crotalus (9.3\%). No entanto, em alguns locais da região Norte do país, as serpentes que causam mais acidentes são as do gênero Lachesis. Os dados obtidos neste estudo divergem, também, dos dados coletados em um trabalho de Cunha et al. (2019), no qual mostram que os acidentes ofídicos que mais ocorrem no estado do Piauí acontecem com serpentes do gênero Crotalus (53.6\%), seguido de Bothrops (28.0\%), Micrurus (5.6\%) e Lachesis (0.8\%).

O maior número de acidentes causados pelas serpentes do gênero Bothrops pode estar relacionado com sua maior diversidade e capacidade de adaptar-se a diferentes tipos de ambientes (Barravieira 1999), distribuindo-se por todo o território brasileiro. São animais de hábitos terrícolas, sendo encontrados em zonas rurais, matas e áreas cultivadas, locais estes propícios para a sua alimentação. Além disso, essas serpentes apresentam um comportamento mais agressivo, atacando rapidamente as pessoas (Pinho \& Pereira 2001; Lira-da-Siva et al. 2009). Os dados revelam ainda que o número de casos ignorados, cerca de $24 \%$ (673), pode resultar da deficiência no atendimento, seja pela ausência do animal ao posto de atendimento ou mesmo pela identificação errônea dos ofídios por parte dos médicos (Bernarde \& Gomes 2012).

As serpentes do gênero Bothrops apresentam uma peçonha com importantes atividades fisiopatológicas (atividade fibrinogenolítica, fibrinolítica e agregadora de plaquetas), lesões locais, ação proteolítica (destruição dos tecidos), ação coagulante, auxilia na liberação de substâncias hipotensoras e provoca lesões na membrana basal dos capilares por ação de hemorraginas (ação hemorrágica). Quando filhote, a ação coagulante predomina e quando adulto predomina a ação proteolítica. Vale ressaltar que a quantidade de peçonha inoculada depende do tamanho da serpente e se ela atacou alguma presa recentemente (Ribeiro \& Jorge 1997; Pinho \& Pereira 2001).

Neste estudo foi observado o registro de acidentes com serpentes do gênero Lachesis (sucucucu-pico-de-jaca), caso observado também por Cunha et al. (2019). No entanto, sua presença no Brasil permeia o bioma Mata Atlântica, onde é encontrada desde o Estado do Ceará até Rio de Janeiro, Amazônia e Norte do Mato Grosso (Almeida et al. 2007; Bernarde 2011, 2019), sugerindo a identificação errônea do animal por parte da população ou da equipe médica, ou o não registro de ocorrência desse gênero para o estado do Piauí, uma vez que, não há estudos que comprovem sua ocorrência para o estado.

Os municípios com maior coeficiente médio de incidência, segundo o Instituto Brasileiro de Geografia e Estatística (IBGE 2017), trabalham com a produção de feijão, mandioca, milho e mamona, além da castanha de caju. Essas atividades no campo podem estar relacionadas com a frequente ocorrência de acidentes com serpentes, uma vez que, é nesse ambiente que esses animais podem ser encontrados. Esses dados corroboram com Lira-da-Silva et al. (2009) em seu estudo sobre Nordeste brasileiro, ao afirmar que Lagoa do Barro do Piauí e Dom Inocêncio apresentam as maiores incidências. Além destes municípios, Lira-da-Silva et al. (2009) registraram maiores incidências também para os municípios de Santana do Piauí e São José do Peixe. Os três municípios com maiores coeficientes de incidência estão localizados nas Microrregiões do Alto Médio Canindé e São Raimundo Nonato, ambas situadas na Mesorregião do Sudeste Piauiense, região semiárida do Piauí.

\section{Conclusão}

Existem poucos trabalhos sobre acidentes ofídicos no estado do Piauí, o que dificulta uma melhor discussão dos dados apresentados. É importante a realização de novos trabalhos que mostrem a realidade do Piauí em relação aos acidentes com serpentes, ressaltando as possíveis causas relacionadas a esses acidentes e as maneiras de prevenção. Devido a uma grande frequência de pessoas que trabalham e/ou moram na zona rural, o encontro e acidente com esses 
animais se torna inevitável, principalmente nos períodos de maior atividade agrícola. A disponibilização de uma educação ambiental para os trabalhadores iria ajudar a minimizar a ocorrência desses acidentes e a ensinar os mesmos os procedimentos corretos para agir quando encontrassem esses animais.

É importante ressaltar que o número de acidentes pode ser ainda maior, uma vez que muitos envolvidos não procuram por atendimento especializado, seja pela crença nos meios naturais alternativos ou até mesmo pela dificuldade de acesso aos serviços médicos. Outro quesito importante é a melhoria e qualificação dos profissionais de saúde nos diversos estágios de atendimento ao paciente, seja pela identificação correta das espécies de serpentes ou pelo preenchimento adequado das fichas de notificação. Estes são de fundamental importância para o prognóstico dos casos e para o desenvolvimento de ações efetivas por parte das autoridades.

Mesmo com a realização de vários estudos que mostram a situação da população brasileira com relação aos acidentes com animais peçonhentos, ainda é escassa ações preventivas por parte das autoridades. Essas ações seriam muito importantes para a diminuição dos acidentes causados por serpentes, enfatizando as medidas preventivas como usos de EPIs (Equipamentos de proteção individual) por parte dos agricultores e outras pessoas em contato direto com o hábitat natural desses animais. Além disso, enfatizar as medidas necessárias a serem tomadas em casos desses acidentes, uma vez que muitas pessoas fazem uso de práticas como torniquetes no local da picada, lavar com querosene, tomar cachaça para cortar o efeito da peçonha, entre outras. Práticas essas que muitas vezes só dificultam o tratamento do paciente e chegam até mesmo a levarem a óbito devido a não procura pelo atendimento médico.

\section{Referências}

Albuquerque H.N., Costa T.B.G. \& Cavalcante M.L.F. (2005) Estudo dos acidentes ofídicos provocados por serpentes do gênero Bothrops notificados no Estado da Paraíba. Revista de Biologia e Ciências da Terra, 5(1): 1-7.

Albuquerque P.L.M.M., Junior G.S., Jacinto C.N., Lima C.B., Lima J.B., Veras M.S.B. \& Daher E.F. (2013) Epidemiological profile of snakebite accidents in a metropolitan area of Northeast Brazil. Revista do Instituto de Medicina Tropical de São Paulo, 55(5): 347-51. http://dx.doi.org/10.1590/S0036-46652013000500009

Almeida A.P., Abe A.S., Gasparini J. \& Argôlo A.J.S. (2007) Os répteis ameaçados de extinção no Estado do Espírito Santo (p. 65-74). In: Passamani M. \& Mendes SL. (Orgs). Espécies da Fauna Ameaçadas de Extinção no Estado do Espírito Santo. Vitória: Instituto de Pesquisas da Mata Atlântica. 140 p.

Barravieira B. (1999) Venenos: aspectos clínicos e terapêuticos dos acidentes por animais peçonhentos. Rio de Janeiro: EPUD. 411 p.

Bernarde O.S \& Gomes J.O. (2012) Serpentes peçonhentas e ofidismo em Cruzeiro do Sul, Alto Juruá, Estado do Acre, Brasil. Acta Amazonica, 42(1): 65-72. http://dx.doi.org/10.1590/S004459672012000100008

Bernarde P.S. (2011) Changes in the Brazilian Poisonous Snake Classification and their Implications in the Medical Literature. Gazeta médica da Bahia, 81(1): 55-63.

Bernarde P.S. (2019) Acidentes ofídicos. Apostila do Laboratório de herpetologia. Centro Multidisciplinar, Campus Floresta, UFAC-AC. Disponível em: http://www.herpetofauna.com.br/OfidismoBernarde.pdf (Acesso em 23 de junho 2019).

Bochner R. \& Struchiner C.J. (2003) Epidemiologia dos Acidentes Ofídicos nos últimos 100 anos no Brasil: uma revisão. Caderno de Saúde Pública, 19(1): 7-16. http://dx.doi.org/10.1590/S0102311X2003000100002

Bochner R. \& Struchiner C.J. (2004) Aspectos ambientais e socioeconômicos relacionados à incidência de acidentes ofídicos no Estado do Rio de Janeiro de 1990 a 1996. Caderno de Saúde Pública, 20(4): 976-85. http://dx.doi.org/10.1590/S0102-311X2004000400012 
Bonan P.R.F., Lima J.S., Martelli D.R.B., Silva M.S., Carvalho S.F.G., Silveira M.D., Marques L.G. \& Júnior H.M. (2010) Perfil epidemiológico dos acidentes causados por serpentes venenosas no norte do estado de Minas Gerais, Brasil. Revista Médica de Minas Gerais, 20(4): 503-507.

Borges C.C., Sadahiro M. \& Santos M.C. (1999) Aspectos epidemiológicos e clínicos dos acidentes ofídicos ocorridos nos municípios do Estado do Amazonas. Revista da Sociedade Brasileira de Medicina, 32(6): 637-646. http://dx.doi.org/10.1590/S0037-86821999000600005

Brasil - Ministério da Saúde. (2001) Manual de diagnóstico e tratamento de acidentes por animais peçonhentos. Brasília, DF: Fundação Nacional de Saúde. 120 p.

Brasil - Ministério da Saúde. (2005) Secretaria de Vigilância em Saúde. Guia de vigilância epidemiológica. $6^{\circ}$ edição. Brasília: Ministério da Saúde. 816 p.

Brasil - Ministério da Saúde. (2009) Secretaria de Vigilância em Saúde. Guia de vigilância epidemiológica. $7^{\circ}$ edição. Brasília: Ministério da Saúde. 816 p.

Brito A.C. \& Barbosa I.R. (2012) Epidemiologia dos acidentes ofídicos no Estado do Rio Grande do Norte. ConScientiae Saúde, 11(4): 535-542.

Casais-e-Silva L.L. \& Brazil T.K. (2009) Acidentes elapídicos no Estado da Bahia: estudo retrospectivo dos aspectos o dos aspectos epidemiológicos em uma série de 14 anos (19801993). Gazeta médica da Bahia, 79(Suplemento 1): 26-31.

Cunha V.P., Santos R.V.S.G., Ribeiro E.E.A., Filho A.L.M.M. \& Marques R.B. (2019) Perfil epidemiológico de acidentes com animais peçonhentos no Piauí. Revinter, 12(1): 76-87. https://doi.org/10.22280/revintervol12ed1.399

D’Augustini F.M., Chagas F.B. \& Beltrame V. (2011) Epidemiologia por acidentes com serpentes no município de Concórdia, SC no período de 2007 a 2010. Evidência, 11(1): 51-60.

Feitosa R.F.G., Melo I.M.L.A. \& Monteiro H.S.A. (1997) Epidemiologia dos acidentes por serpentes peçonhentas no Estado do Ceará-Brasil. Sociedade Brasileira de Medicina Tropical, 30(4): 296301. http://dx.doi.org/10.1590/S0037-86821997000400004

Gutiérrez J.M. (2011) Envenenamientos por mordeduras de serpientes en América Latina y el Caribe: Una visión integral de carácter regional. Boletín de Malariología y Salud Ambiental, 51(1): 1-16.

Gutiérrez J.M., Theakston R.D.G. \& Warrell D.A. (2006) Confronting the Neglected Problem of Snake Bite Envenoming: The Need for a Global Partnership. PLOS Medicine, 3(6): e150. https://doi.org/10.1371/journal.pmed.0030150

Gutiérrez J.M., Higashi H.G., Wen F.H. \& Burnouf T. (2007) Strengthening antivenom production in Central and South American public laboratories: report of a workshop. Toxicon, 49(1): 30-35. https://doi.org/10.1016/j.toxicon.2006.09.005

Harrison R., Hargreaves A., Wagstaff S.C., Faragher B. \& Lallo D.G. (2009) Snake Envenoming: A Disease of Poverty. PLOS Neglected Tropical Diseases, 3(12): e569. https://doi.org/10.1371/journal.pntd.0000569

IBGE (2017) Produção Agrícola Municipal 2016. Disponível em: https://cidades.ibge.gov.br/brasil/ pi/panorama (Acessado em 16/06/2019).

Lemos J.C., Almeida D., Fook S.M.L., Paiva A.A. \& Simões M.O.S. (2009) Epidemiologia dos acidentes ofídicos notificados pelo Centro de Assistência e Informação Toxicológica de Campina Grande (Ceatox-CG), Paraíba. Revista Brasileira de Epidemiologia, 12(1): 50-59. http://dx.doi.org/10.1590/S1415-790X2009000100006

Lima A.C.F.S., Campos C.E.C. \& Ribeiro J.R. (2009) Perfil epidemiológico dos acidentes ofídicos do Estado do Amapá. Sociedade Brasileira de Medicina Tropical, 42(3): 329-335. http://dx.doi.org/10.1590/S0037-86822009000300017

Lira-da-Silva R.M., Mise Y.F., Brazil T.K., Casasi-e-Silva L.L. \& Carvalho F.M. (2009) Morbimortalidade por ofidismo no Nordeste do Brasil (1999-2003). Gazeta médica da Bahia, 79(Suplemento 1): 21-25.

Lucas E.P.R. (2009) Estudo interlaboratorial para o estabelecimento do veneno botrópico e do soro antibotrópico de referência nacional. Dissertação de Mestrado, Programa de PósGraduação em Vigilância Sanitária. Fundação Oswaldo Cruz, Instituto Nacional de Controle de Qualidade em Saúde, Rio de Janeiro. 
Oliveira N.R., Sousa A.C.R., Belmino J.F.B., Furtado S.S. \& Leite R.S. (2015) The epidemiology of envenomation via snakebite in the State of Piauí, Northeastern Brazil. Sociedade Brasileira de Medicina Tropical, 48(1): 99-104. http://dx.doi.org/10.1590/0037-8682-0173-2014

Pinho F.M.O. \& Pereira I.D. (2001) Ofidismo. Associação Médica Brasileira, 47(1): 24-29.

Pinho F.M.O., Oliveira E.S. \& Faleiros F. (2004) Acidente ofídico no estado de Goiás. Associação Médica Brasileira, 50(1): 93-96.

Ribeiro L.A. \& Jorge M.T. (1997) Acidente por serpentes do gênero Bothrops: série de 3.139 casos. Sociedade Brasileira de Medicina Tropical, 30(6): 475-480. http://dx.doi.org/10.1590/S003786821997000600006

Rojas C.A., Gonçalves M.R. \& Almeida-Santos S.M. (2007) Epidemiologia dos acidentes ofídicos na região noroeste do estado de São Paulo, Brasil. Revista Brasileira de Saúde e Produção Animal, 8(3): 193-204.

Saraiva M.G., Oliveira D.S., Filho G.M.C.F., Coutinho L.A.S.A. \& Guerreiro J.V. (2012) Perfil epidemiológico dos acidentes ofídicos no Estado da Paraíba, Brasil, 2005 a 2010. Epidemiologia e Serviços de Saúde, 21(3): 449-456. http://dx.doi.org/10.5123/S167949742012000300010

SINAN (2017) Acidentes por animais peçonhentos. Disponível em: http://tabnet.datasus.gov.br/cg i/deftohtm.exe?sinannet/cnv/animaispi.def (Acessado em 09/09/2019).

Sousa R.S., Costa K.M.F.M., Câmara I.M.B., Oliveira G.B., Moura E.S.R., Fonseca Z.A.A.S., Moreira J.O. \& Leite A.I. (2013) Aspectos epidemiológicos dos acidentes ofídicos no município de Mossoró, Rio Grande do Norte, no período de 2004 a 2010. Revista Patologia Tropical, 42(1): 105-113.

Tavares A.V. (2016) Estudo epidemiológico e clínico dos casos de acidentes ofídicos no Estado do Rio Grande do Norte. Dissertação de Mestrado, Programa de Pós-Graduação em Ciências Naturais e Biotecnologia. Universidade Federal de Campina Grande, Campina Grande, Paraíba.

Waldez F. \& Vogt R.C. (2009) Aspectos ecológicos e epidemiológicos de acidentes ofídicos em comunidades ribeirinhas do baixo rio Purus, Amazonas, Brasil. Acta Amazonica, 39(3): 68192. http://dx.doi.org/10.1590/S0044-59672009000300025 